\title{
CAMINAR ENTRE LUCHAS Y PRUEBAS: FUNCIONES PARADÓJICAS DE LA ENACCIÓN DEL ESPÍRITU SANTO EN EL CULTO PENTECOSTAL
}

\author{
WALKING THROUGH STRUGGLES AND TRIALS: PARADOXICAL FUNCTIONS \\ OF THE ENACTION OF THE HOLY SPIRIT IN PENTECOSTAL WORSHIP
}

\author{
Rodrigo Moulian ${ }^{1}$, Iván Oliva ${ }^{2}$ y Sergio Toro ${ }^{2}$
}

\begin{abstract}
El presente artículo muestra el carácter paradójico de las funciones que asume la enacción del "Espíritu Santo" en el culto pentecostal, como medio para administrar la aflicción y fuente para socializar la resignación frente a las causas de la misma. Por enacción entendemos aquí el proceso en que la acción humana se impregna de sentido, trayendo a la vida las distinciones cognitivas. En el caso de la liturgia pentecostal, esta noción comprende a los recursos por los que el "Espíritu Santo" se hace presencia encarnada y actuante en el culto, produciendo experiencias numinosas que inspiran admiración y "temor a Dios" entre los fieles. Nuestro análisis muestra cómo la estructura de la comunicación ritual, caracterizada por la "retórica de la presencia", produce una experiencia somatognósica, en la que las concepciones sagradas se viven "en carne propia". Los pentecostales denominan a esto "el sello del Espíritu", lo que constituye uno de los rasgos distintivos de su religiosidad. El estudio del contexto de la comunicación ritual, de las situaciones sociobiográficas que enfrentan los fieles y los tópicos dominantes de la trama litúrgica, muestra que una de sus principales funciones psicosociales es el manejo de las aflicciones humanas. El culto ayuda a mitigar los estados angustiosos derivados de las crisis vitales, pero paradojalmente, en ocasiones, también se encarga de reproducirlos, generando un círculo causal donde aflicción y carisma se retroalimentan y potencian.
\end{abstract}

Palabras claves: Pentecostalismo, carisma, enacción, aflicción, sobreadaptación.

This paper shows the paradoxical nature of the functions assumed by the enaction of the "Holy Spirit" in Pentecostal worship, as a means to manage grief and as a way to socialize resignation to what causes it. By enaction, we mean the process by which human action is imbued with meaning by bringing forth cognitive distinctions to life. Our analysis shows how ritual communication structure, characterized as "rhetoric of presence", produces a bodily presence in which sacred representations find life in "their own flesh". Pentecostals name this "the seal of Spirit", which constitutes one of the distinctive features of their religiosity. The study of context in ritual communication, worshipper's socio-biographical situations, and dominant topics in ritual plot, shows that one its main socio-psychological functions is to cope with human affliction. Worship helps to lessen states of anguish caused by life crisis, but also, and paradoxically, sometimes, reproduces it, making a causal circle where both affliction and charisma are feedbacked and powered each other.

Key words: Pentecostalism, charisma, enaction, affliction, over-adaptation.

La religiosidad pentecostal se distingue y especifica por la enacción del "Espíritu Santo" en el espacio ritual. Es decir, por procesos de cognición encarnada, de estructura autopoiética y cualidades transformadoras que proveen una experiencia sensible de las concepciones del "Espíritu" que, de este modo, se pone en acción y "obra" sobre la vida de los participantes. La mediación ritual, no obstante, tiene rasgos paradójicos. Si bien estos procesos de enacción producen en los fieles efectos terapéuticos y revivificantes, sirven también a la legitimación de un sistema ideológico que promueve el quietismo social y con ello favorecen la reproducción de las condiciones que generan crisis vitales y estados de aflicción. Sus prácticas religiosas resultan transformadoras a nivel personal, pero conservadoras en lo social, de modo que los fieles encuentran en el culto un lugar para "liberarse" momentáneamente y luego reinsertarse en los contextos que generan el malestar.

Las nociones de "luchas" y "pruebas" expresan, en la cultura pentecostal, la doble tensión que habitualmente enfrentan los fieles, amenazados por un mundo que se les presenta adverso y ajeno,

\footnotetext{
1 Instituto de Comunicación Social, Universidad Austral de Chile, Valdivia, Chile. rmoulian@hotmail.com

2 Instituto de Filosofía y Estudios Educacionales, Universidad Austral de Chile, Valdivia, Chile. ivanoliva@uach.cl; seatoro@gmail.com
} 
y exigidos por un "Espíritu" celoso, que somete a examen su fidelidad en las contrariedades. "Luchas" y "pruebas" son hitos que describen el tránsito existencial de los fieles en un ciclo que los conduce recurrentemente al culto y sirve a su reproducción. Para hacer frente a unas y sobrellevar las otras, el pentecostalismo ofrece la fuerza protectora y restauradora del "Espíritu Santo", expuesta en un magisterio de "obras", "prodigios" y "señales sobrenaturales", que muestran la vigencia del misterio de Pentecostés. De acuerdo con el libro Hechos de los Apóstoles (2:1-11), este día los discípulos de Cristo recibieron la visitación del Espíritu Santo, que se les apareció en forma de llamas de fuego y se manifestó en ellos a través de la glosolalia o "hablar en lenguas extrañas". El evangelismo carismático, que se inspira en este acontecimiento, nos sitúa en el campo fenomenológico de la pneumatología y nos plantea el problema de la eficacia del símbolo y sus distintas expresiones históricas (Figura 1).

\section{Confesiones de Teoría y Método}

Nuestro trabajo se inspira en el paradigma de las mediaciones sociales (Martín Serrano 1977), que procura el estudio de las articulaciones de las instituciones humanas en diversos niveles de realidad. En la forma que lo asumimos (Moulian 2012), ello supone en primer momento el despliegue de un esfuerzo comprensivo de las lógicas culturales y las experiencias de los actores en sus propios términos, lo que se caracteriza como la perspectiva emic (Pike 1967). En segundo lugar, el análisis de las determinaciones e intervenciones funcionales de sus prácticas en los diversos dominios sistémicos donde operan, es decir, una perspectiva explicativa o etic. La teología se lee aquí, por lo tanto, en su relación con los campos biológico, psicológico y social.

En el caso del estudio de las mediaciones rituales, esto implica la atención de los procesos de comunicación litúrgicos, con sus componentes narrativos y retóricos, de los contextos socioculturales donde éstos operan y las situaciones que enfrentan sus participantes. Dicho de otro modo, se trata de atender tanto a las características internas del rito como a las condiciones sobre las cuales se desarrolla la acción simbólica, para ver sus articulaciones e implicaciones mutuas.

Como método de investigación empleamos el estudio etnográfico de caso. Durante tres años

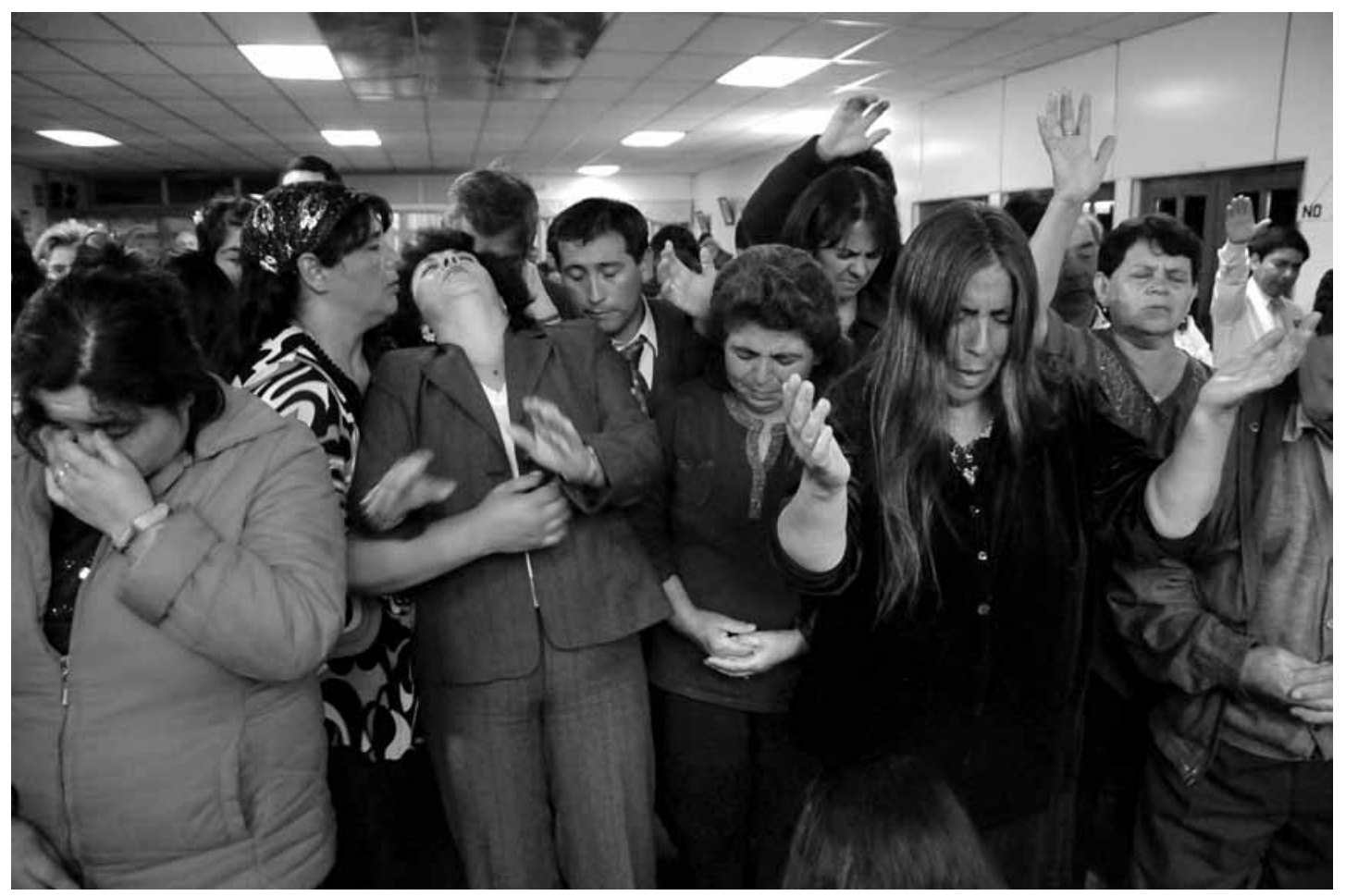

Figura 1. "Quebrantamiento espiritual”, comienzo de la enacción del Espíritu Santo.

"Spiritual brokenness", beggining of the Holy Spirit's enaction. 
hemos seguido a un Ministerio Evangelista de Sanidad y Liberación que realiza su trabajo de manera itinerante entre la XIV y X regiones del sur de Chile. Éste, mensualmente, organiza vigilias en distintas localidades donde convergen personas de múltiples denominaciones pentecostales, lo que nos ha permitido conocer diferentes sensibilidades dentro de este movimiento. De modo complementario, realizamos trabajo de campo en dos iglesias establecidas de Valdivia. Estas congregaciones han sido seleccionadas por el carácter carismático de su religiosidad, donde se expresa nítidamente el paradigma pentecostal.

El ejercicio de observación participante se ha desarrollado por investigadores con distinta formación disciplinaria, en campos como la antropología, la comunicación, la biología y las ciencias de la motricidad. Ello ha permitido la focalización de la atención en diversas dimensiones de la acción ritual, como el simbolismo, la retórica, la somática y la kinésica. Las prácticas cúlticas a las que asistimos fueron registradas en formato audiovisual, fotográfico y auditivo, lo que ha permitido la revisión, discusión y análisis de estos datos desde un posicionamiento interdisciplinar. Las acciones y experiencias rituales que se recogen allí han sido contextualizadas y cualificadas a través de entrevistas en profundidad a los participantes. Éstas han sido conducidas con el propósito de especificar el sentido de los actos litúrgicos, las condiciones y circunstancias biográficas de los asistentes y acceder a sus vivencias personales. A fin de ganar profundidad y precisión en los datos, durante las entrevistas hemos mostrado a nuestros informantes los materiales de registro de los cultos. Los resultados emergentes en estas conversaciones nos han permitido orientar nuestra atención por medio de la práctica de observación dirigida y entrevistas focalizadas.

\section{Antecedentes del Pentecostalismo}

En Chile, el pentecostalismo es el movimiento religioso que muestra mayor crecimiento durante el siglo XX, posicionándose como la principal corriente dentro del campo evangélico nacional. Por lo mismo, ha ganado visibilidad y derechos de ciudadanía sociocultural. No en vano el llamado " $T e$ Deum evangélico" se desarrolla en la catedral de la Iglesia Metodista Pentecostal, la primera iglesia histórica de esta denominación y una de las que presenta mayor membresía. El censo estadístico de
1920, el primero que incorpora la variable religiosa, registra una adscripción evangélica de 1,4\% de la población. Para el año 2002, esta cifra se eleva al $15,14 \%$ de los chilenos, lo que corresponde a un universo de 1.699 .725 personas. Los investigadores del fenómeno coinciden en destacar el predominio pentecostal dentro de este espectro religioso. Así lo consignan trabajos como los de Bothner (1994), Corvalán (2009), Canales et al. (1991), Ossa (1991), Parker (1993), Poblete y Galilea (1984), Spoerer (1984) y Tennekes (1985), cuyas estimaciones sobre el rango de representatividad del pentecostalismo varían entre el 70 y el $95 \%$ de este universo. Dicho de otro modo, aun si nos situamos en los cálculos más moderados, hoy es pentecostal uno de cada diez chilenos.

El pentecostalismo constituye un movimiento religioso "de salvación" que rebasa los límites institucionales y se distingue por un modo particular de vivir la fe. No obstante, su crecimiento ha ido de la mano de un grado importante de diversificación interna que obliga a tener cautela con las generalizaciones. Orellana (2011) identifica cuatro tipos de manifestaciones organizacionales como principales formas de expresión de éste: (a) grandes iglesias nacionales institucionalizadas; (b) iglesias de tamaño medio de origen misionero o nacional y presencia zonal; (c) cultos de la prosperidad de origen brasilero; (d) pequeñas iglesias locales y ministerios autónomos. Es en este último tipo de iglesias donde se circunscribe nuestro trabajo. Al respecto, es importante advertir que el proceso de institucionalización de las iglesias nacionales históricas ha producido una burocracia eclesial y se encuentra acompañado de una rutinización del carisma, de modo que algunas de sus congregaciones viven un pentecostalismo doctrinal o nominal más que experiencial. No obstante, las manifestaciones espirituales continúan cultivándose en las pequeñas iglesias locales, que reproducen el modelo carismático. Es esta forma de religiosidad la que nos interesa estudiar, porque constituye la matriz de la identidad pentecostal y nos plantea con mayor nitidez el problema de su eficacia simbólica.

El perfil sociobiográfico de los fieles se muestra aquí como un factor relevante. El crecimiento del pentecostalismo se concentra, fundamentalmente, en los sectores populares. Su religiosidad ha sido caracterizada como "cultura de las clases bajas" (Willems 1967), un "refugio de las masas" (Lalive 1968), "una respuesta de fe desde la marginalidad" 
(Mosher 1995), "un mecanismo de empoderamiento de los impotentes" (Sjørup 2002). Este rasgo constituye una constante en los diversos contextos socioculturales donde ha fructificado el movimiento. De allí que Hollenweger (1969) lo caracterice como una religión de los "desheredados". Al respecto, resulta sugerente la lectura de Chesnut (1997), quien sostiene que el pentecostalismo es una respuesta a los efectos patogénicos de la pobreza. Un reciente estudio de Corvalán (2009) sobre el crecimiento de esta denominación en nuestro país, desde las fuentes censales, ratifica que éste se expande en el último período en las comunas que muestran menor escolaridad, entre personas con menor calificación para el trabajo, en áreas con menor índice de desarrollo humano y tiene mayor representación entre la población más vulnerable del país: personas con algún tipo de discapacidad, indígenas y mujeres. Nuestra experiencia de investigación confirma estos antecedentes. Hemos encontrado que el pentecostalismo se erige como la principal adscripción religiosa en algunas comunidades mapuches-williches (Moulian 2012) y constatado un componente predominante de género femenino en sus cultos (Moulian 2010). Resulta relevante preguntarse, entonces, por las cualidades del culto que resultan atractivas y satisfactorias para estos tipos de destinatarios, marcados por la subalteridad, la marginalidad y la deprivación.

Con variantes y combinaciones diversas, las propuestas explicativas del crecimiento del pentecostalismo chileno exponen, de modo preponderante, argumentos funcionalistas y culturalistas, que ponen de relieve las condiciones o características sociales y simbólicas del contexto donde éste se produce. El evangelismo carismático ha sido considerado una respuesta adaptativa a la desestructuración de la sociedad tradicional (Willems 1967), el carácter anómico de una sociedad en transición (Lalive 1968), las condiciones de explotación y exclusión propias del capitalismo moderno (Parker 1993), ante las que viene a satisfacer las necesidades de integración, de provisión de sentido y sirve de mecanismo de compensación simbólica frente a la miseria. Según Bastian (1997), constituye un mecanismo de defensa y repliegue comunitario ante el reflujo de las ideologías, que permite a los sectores marginales reestructurarse. Su selección se debería a las relaciones de afinidad con la religiosidad popular (Tennekes 1985; Willems 1967) o con las religiones indígenas de procedencia de los neófitos (Foerster 1993; Guerrero 1994) que, al igual que éste, ponen énfasis en los aspectos instrumentales, tienen un carácter taumatúrgico o énfasis pneumatológico. Como apunta Bastian (1997:209), "el constante recurso al triple ejercicio de la glosolalia, de la taumaturgia y del exorcismo, perpetúan el universo cultural de las mayorías". Lo que predomina en el pentecostalismo, sostiene este autor, "es la fuerza emocional de la religión y no su fuerza cognitiva y crítica" (Bastian 1997:213).

Por lo mismo, consideramos que para entender el éxito del pentecostalismo es necesario avanzar en el conocimiento de los mecanismos sobre los que descansa la eficacia simbólica en el culto, para luego estudiar sus funciones. Dicho de otro modo, planteamos que no son el nomos, la ética o las relaciones de solidaridad las que explican el crecimiento del pentecostalismo, ya que estos elementos se encuentran también en otras iglesias cristianas. A la inversa, es el tipo de experiencia religiosa que el pentecostalismo ofrece la que produce una comunidad provista de nomos, ética y relaciones de solidaridad forjadas bajo "el sello del Espíritu". Nuestra atención aquí se concentrará en la caracterización y análisis de la gestión de esta experiencia y en las funciones que esta desempeña.

\section{Enacción del Espíritu Santo}

La identidad pentecostal se funda en la enacción del "Espíritu Santo", en tanto se considera que es la capacidad de los fieles de entrar en un contacto directo y sensible con éste lo que constituye y define a este movimiento. El término "enacción" proviene del verbo inglés to enact que, en el campo de las artes escénicas, significa "representar". Una segunda acepción del mismo es "llevar a cabo". El campo semántico de esta palabra se desplaza entre la actuación y las propiedades realizativas. Bruner (1968) la emplea para designar al aprendizaje fundado en la acción, provisto, por lo tanto, de un carácter sensomotriz. Como concepto, la "enacción" ha devenido en una de las nociones paradigmáticas de la epistemología constructivista. Varela et al. (1992) y Varela $(2000,2002)$ lo usan para designar el proceso de emergencia del conocimiento enraizado en el cuerpo y en la historia social del sujeto cognoscente. De acuerdo con estos autores, conocer no consiste en representarse un mundo independiente y preexistente, sino en producir una imagen de él. En otras palabras, la 
cognición es interpretada como un proceso enactivamente encarnado de co-determinación entre lo interno y lo externo, a la vez de ser inseparable de operar del organismo como un todo. Como apunta el clásico aforismo de Maturana y Varela (1994 [1984]:13), "conocer trae un mundo a la mano". Es lo que sucede en el culto pentecostal, donde las concepciones teológicas carismáticas cobran vida en la experiencia corporalizada de los fieles que se gesta en el marco de una acción relacional aprendida (Toro 2011). En la acción ritual, los fieles sienten la presencia y experimentan las obras del "Espíritu Santo" en sus vidas. Como argumentaremos, ello es resultado de la vibrante trama comunicativa que despliega la liturgia pentecostal, a través de la que se produce la enacción espiritual.

$\mathrm{Al}$ interior del culto carismático, el "Espíritu Santo" se configura en la experiencia de los fieles, encarnado en las señales que emergen de la propia corporalidad, las que se impregnan de sentido en la enunciación litúrgica. Dicho de otro modo, el "Espíritu Santo" es enactuado en el culto. Este proceso posee una estructura causal recursiva y autorreferente, puesto que la acción ritual opera tanto como dispositivo de gestión de la experiencia cuanto como marco de referencia para la interpretación de aquella. De este modo, las concepciones distintivas de la pneumatología pentecostal cobran vida y se experimentan "en carne propia". Este anclaje corporal es la base para el testimonio y reivindicación de un Dios "vivo" y "real" que caracteriza el discurso pentecostal. Las propiedades terapéuticas y reconfortantes de los somatotropismos que gestiona el ritual, activadas en el contexto de crisis vital de los participantes, son una de las claves del éxito de la oferta religiosa, promovidas como obras del ministerio espiritual.

\section{Comunicación y Encarnación}

En nuestra perspectiva, la eficacia simbólica que exhibe el pentecostalismo descansa en los mecanismos de la comunicación ritual, que emplea principios que por sí mismos resultan productivos. Entre éstos se cuentan: (i) la implicación pragmática de los participantes, que actúan como protagonistas de la acción ritual dirigida a la "administración del Espíritu Santo" en sus propias vidas; (ii) el empleo de recursos comunicativos multimediales que envuelven y comprometen corporalmente a los asistentes en la ejecución del culto; (iii) el despliegue de una estructura dramática realizativa, biográficamente anclada, sobre la base de la enacción de las distinciones simbólicas por parte de los fieles; (iv) el empleo de una "retórica de la presencia", transversal a la acción ritual, que identifica las señales sobrenaturales en el desempeño comunicativo de los participantes y los efectos corporales que la propia comunicación ritual produce sobre éstos. Así, los procesos de transformación de las emociones, los cambios en los estados de conciencia y la modificación de las manifestaciones sintomáticas producidas en la dinámica del culto se interpretan como evidencias de la acción del "Espíritu Santo".

El caso pentecostal muestra, de manera ejemplar, cómo los procesos de producción social de la corporalidad desempeñan un papel central en la construcción social de la realidad, de modo que ésta se funda a la vez que decanta en el cuerpo. La noción de "enacción" permite comprender la circularidad de esta articulación entre el conocimiento como experiencia corporizada y construcción social. Ésta refiere al proceso en el que la acción adquiere significado en la interacción humana, de modo que las distinciones culturales cobran vida y se "naturalizan", es decir, se hacen parte del sentido común vivido. En el pentecostalismo, este proceso se muestra de un modo particularmente nítido, ya que la invocación a lo sagrado parte de la situación sociobiográfica de los recurrentes, de modo que la religiosidad se fundamenta en su experiencia. Como señalamos, el primer principio comunicativo sobre el que se construye la eficacia simbólica es la implicación pragmática de los participantes del culto. La comunicación litúrgica se encuentra diseñada para proveer una experiencia personalmente transformadora. En tanto, la doctrina socializa una ética social que favorece la adaptación o sobreadaptación a los contextos donde éstos se desenvuelven cotidianamente. Su eficacia en lo primero sirve para la legitimación de las representaciones y valores que hacen posible lo segundo. Estos preceptos se encargan, a su vez, de reproducir las condiciones que hacen necesario seguir yendo al culto.

\section{Niveles de Análisis Funcional}

El ciclo en que se ven envueltos los participantes del culto va desde la "liberación" ritual a la resignación social. Si bien los servicios religiosos pentecostales muestran virtudes terapéuticas, su doctrina promueve una actitud conservadora incluso 
frente a los componentes patológicos del orden social. La reproducción de ellos refuerza la necesidad de ir al culto en la búsqueda compensatoria de la unción del "Espíritu Santo". Al respecto, resulta ilustrativo el testimonio que nos entrega un pastor:

Hay mucha mujer que sufre. Vienen heridas, llegan destruidas a buscar sanidad del alma. Mujeres que han sido violadas, mujeres a las que el marido con alcohol en la sangre las obliga a tener sexo, y como son cristianas y le temen a Dios, soportan. Niñas jóvenes que quieren irse de la casa, cansadas del maltrato psicológico que les dan sus padres. Esa violencia mata, va hiriendo y se acumula. Mujeres deprimidas por falta de cariño y comprensión. Mujeres evangélicas que son maltratadas, a las que el marido hasta las golpea después de una vigilia. Ellas soportan y están autoconvencidas que éstas son pruebas por parte de Dios. Esa gente que está muy enferma del alma viene a la vigilia, llora, se liberta. El Señor trata sus corazones, les da risa santa que es la mejor terapia, pero después vuelven a su hogar y se encuentran con la misma realidad. Lo ideal sería otro ambiente, para mantener la bendición que Dios les ha dado. El problema es que después retornan a su hábitat y eso le quita efecto a lo logrado, volviendo por los mismos caminos.

Las mediaciones que ofrece el culto presentan un carácter contrastante con las de su ética social. Se deben distinguir, por lo tanto, dos niveles de análisis funcional del pentecostalismo: uno psicosocial y otro sociocultural, cuyos efectos resultan paradójicos, pero se encuentran articulados al servicio de la reproducción de este sistema religioso. Como expondremos, son las características comunicativas de la liturgia, que modela los procesos de enacción del Espíritu Santo, la clave del éxito de este movimiento. La experiencia religiosa es el sustento de su doctrina y ética.

\section{Señales de la Presencia}

En términos psicosociales, el culto pentecostal se puede definir como un ritual de aflicción (Alexander 1989). La experiencia litúrgica es un medio para manejar los estados angustiosos. Su llamado se dirige, especialmente, a los que sufren a causa de la precariedad económica, soledad o enfermedades, a quienes invita a acercarse a un Padre Todopoderoso, calificado como el "Gran Consolador". El contexto sociobiográfico de los fieles que entrevistamos confirma este perfil carenciado, donde encontramos testimonios de violencia doméstica, experiencias carcelarias, problemas de salud mental. En sus historias de vida se vislumbra una correlación tendencial entre la magnitud de los dramas personales y la intensidad de las manifestaciones carismáticas. A mayor sufrimiento, mayor la necesidad de buscar consuelo en el "Espíritu", que se hace presente de manera sensible en sus cuerpos.

El servicio religioso pentecostal es un espacio catártico. Los concurrentes asisten a él para dejar sus "cargas" en las manos de Dios, mediante la plegaria y el llanto. Los pentecostales denominan a esto el "quebrantamiento", pues supone el reconocimiento de la necesidad de Dios y la desactivación de los mecanismos de defensa y control de las emociones. Esto ocurre, habitualmente, en la oración inicial en la que los fieles presentan sus necesidades y requerimientos. La evocación de las situaciones de crisis vital activa las emociones angustiosas asociadas a estas y sus correspondientes mecanismos fisiológicos de respuesta. La anamnesis afectiva opera desde la memoria somática, de modo que los participantes reviven su dolor en el culto. El llanto, que le sigue, constituye un mecanismo homeostático natural, destinado a descargar a través de las lágrimas los niveles de cortisol generados por el cerebro en las situaciones de estrés. Esta descarga produce una sensación de liberación y paz interior. Para los pentecostales es una señal de que el "Espíritu Santo" comienza a obrar en la vida de los concurrentes. Así, la experiencia gestada por el propio proceso ritual se transforma en una pieza de su trama retórica que, como advierte Poewe (1989) para el caso del catolicismo carismático, tiene una estructura metonímica, pues se encuentra dominada por la idea de contigüidad. "Quebrantarse" es una de las formas de enacción del Espíritu.

El ejercicio del canto congregacional es el medio para conducir a los participantes desde la catarsis al "avivamiento" y desde el "quebrantamiento" al "gozo". Una de las propiedades más notables de la música es la de la sincronización somática, consistente en la modificación del biorritmo y los estados anímicos asociados a ellos. Esto se verifica por el llamado "enganche" o "rapto rítmico" (Becker 
2005; Merker et al. 2009), como se denomina la tendencia instintiva a seguir el ritmo musical con movimientos del cuerpo, lo que se explica por la proximidad e inervación entre la corteza auditiva y motriz (Zatorre et al. 2007). Los temas de tempo rápido, también llamado allegro, producen una activación de la corporalidad e inducen estados anímicos positivos. En tanto, los de tempo lento tienden al apaciguamiento. Si bien las iglesias pentecostales administran un amplio repertorio, adecuado para las diversas fases del culto, en la liturgia carismática predominan los temas rápidos y alegres destinados a animar a la congregación. El culto se vuelve un espacio celebrativo, donde los concurrentes viven la fiesta de Pentecostés. El entusiasmo puesto por los participantes en la ejecución de los temas es tenido como señal de "avivamiento espiritual". La experiencia revivificante y las sensaciones de energización y bienestar que ello suscita, denominadas "gozo en el espíritu", también se tienen como señal inequívoca de la presencia divina. El canto congregacional opera así como otra de las formas de la enacción del "Espíritu".

La presencia divina también se encuentra denotada en los procesos de disociación inducidos por la acción ritual que experimentan los fieles. El concepto de disociación designa a los estados de conciencia marcados por la escisión funcional, la focalización de la atención y la restricción del procesamiento y flujo de la información (Seligman y Kirmayer 2008). Entre sus manifestaciones se encuentran, por ejemplo, el desplazamiento del pensamiento intelectivo hacia una conciencia sensomotriz o bien, por el contrario, la disolución de la imagen corporal, que caracterizan a algunas manifestaciones carismáticas. De allí que en términos fenomenológicos el pentecostalismo sea clasificado como "un ritual de posesión" (Lewis 1989 [1971]) o de "trance" (Bourguignon 1973), pues supone un descentramiento de la identidad y la conciencia de los participantes, que viven experiencias de posesión espiritual o éxtasis religioso.

La llamada "danza en el Espíritu" es un ejemplo de los fenómenos de posesión, pues según las concepciones pneumatológicas pentecostales, los fieles bailan "bajo la cobertura del Espíritu Santo". Habitualmente ésta se despliega en el marco del "avivamiento" musical, durante el que algunos fieles se dejan llevar por el ritmo. Esta práctica supone una focalización de la atención en la propia corporalidad de los ejecutantes, de modo de ajustar los movimientos a la capacidad aeróbica y adecuarlos a los patrones de la música. Los danzantes se ven sumergidos en sus coreografías. En los términos de Bourguignon (1973), se trata de un estado de disociación hiperactivo, pues supone un intenso compromiso kinésico, acompañado por la remisión del pensamiento reflexivo en favor del ejercicio sensomotriz y la conciencia somática, y un vuelco hacia la información propioceptiva en desmedro de la lectura del entorno. De acuerdo con el credo pentecostal, es el "Espíritu Santo" quien toma el control de sus cuerpos. Los fieles declaran sentirse "movidos por una fuerza exterior" que los conduce, impulsados "por una corriente de fuego". La danza es, por lo mismo, un significante de poder.

La caída en el llamado "manto blanco de Jesús" constituye, en tanto, una de las formas de éxtasis místico. Una vía de acceso a éste es el ejercicio de la "oración en el Espíritu", lo que supone un recogimiento en la oración. Quien se dispone a ella habitualmente cierra los ojos y concentra toda su atención en su enunciado, que sigue fórmulas estereotipadas y estructuras iterativas empleadas de manera persistente. Esta práctica se asocia a experiencias como la automatización de la oración, el desarrollo perceptivo de una imagen blanca que cubre a los fieles y la sensación de disolución del cuerpo. En términos neurofisiológicos (Newberg et al. 2001), ello se explica por una caída en la actividad del área de procesamiento de la información somatosensorial ubicada en el lóbulo parietal, producto de la focalización de la atención en la oración. Los pentecostales lo vivencian como una "comunión con el Señor" y "cobertura por el manto blanco", que les produce un "gozo en el Espíritu". La disociación es otra forma de enacción pentecostal.

\section{El Sello del Espíritu}

La dimensión pragmática predomina en el culto. Su enunciación tiene un carácter realizativo, pues administra el poder del "Espíritu Santo" para atender las necesidades de los concurrentes. Aunque en la perspectiva pentecostal éste puede actuar en diversos momentos de la liturgia, el servicio religioso reserva un espacio especialmente diseñado para ello. Es el denominado acto de "unción" o "administración espiritual", donde se realizan las "obras de sanidad" y "liberación divina" mediante un desempeño ritual performativo. Las acciones son dirigidas por los llamados "instrumentos del 


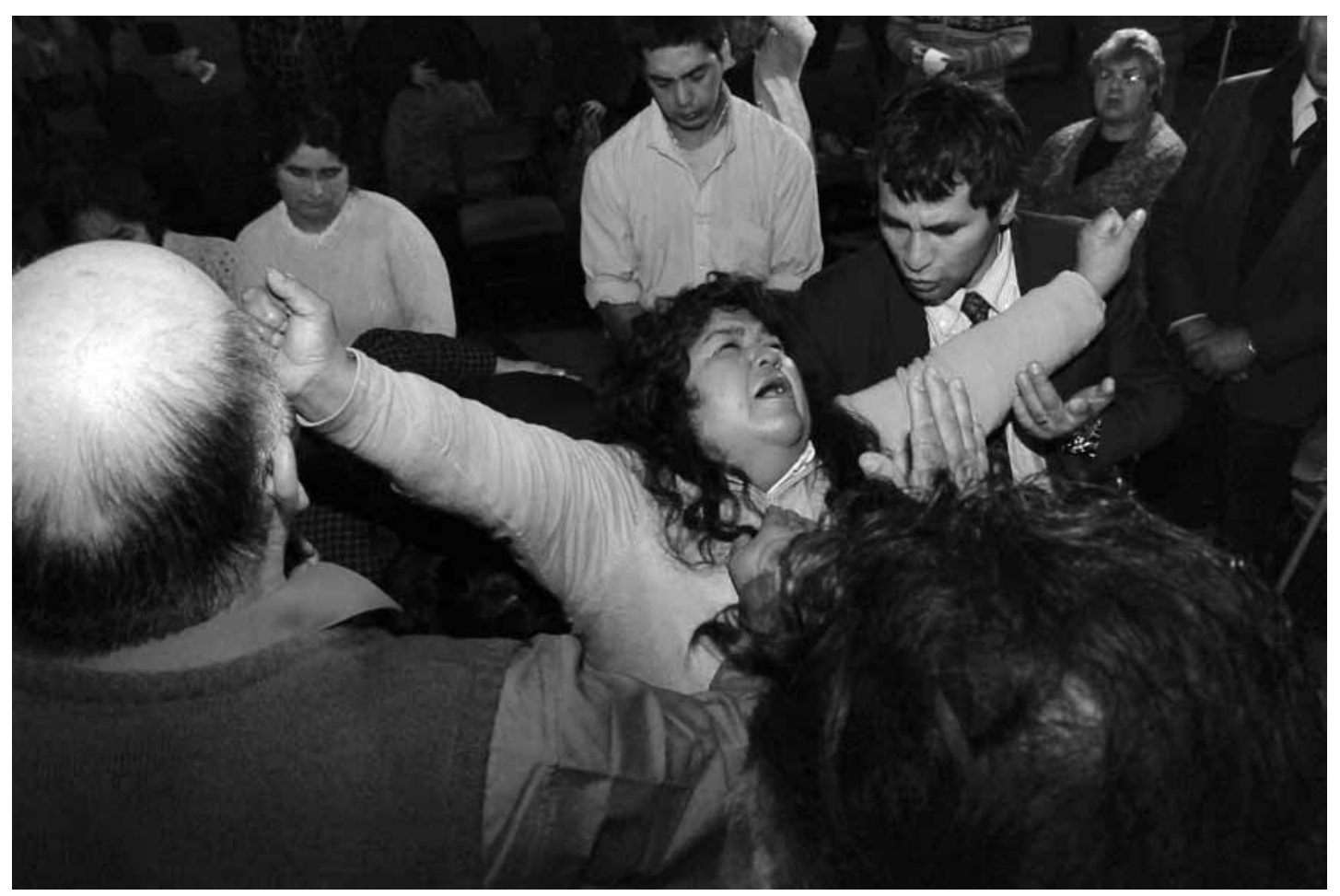

Figura 2. Imposición de manos como "sello del Espíritu Santo".

Laying on of hands as "seal of the Holy Spirit".

Señor", entre los que hay "apóstoles", "evangelistas", "profetas", a quienes se considera depositarios de sus dones divinos, por medio de cuyas manos opera el "poder de Dios", que a través de "sus obras de sanidad" deja su "sello" sobre los fieles (Figura 2).

Estos "instrumentos del Señor" hacen el llamado a los asistentes para que pasen al púlpito quienes se encuentren en condición de necesidad, a fin de recibir la "unción divina". "Usted que se encuentra agobiado, usted allá atrás que está abatido por la enfermedad o el dolor, pase y recibirá bendición", proclaman los agentes rituales en un discurso que suele apelar a los problemas que caracterizan el fondo común de la experiencia humana. Quienes concurren al llamado reconocen públicamente su situación de necesidad, lo que activa nuevamente los estados emotivos que traen consigo al templo. El pentecostalismo trabaja sobre su vulnerabilidad.

El foco de la acción ritual se dirige a la situación biográfica de los solicitantes. Los agentes indagan sobre los requerimientos individuales de cada uno para dirigir su intervención hacia estos intereses particulares. Aproximan sus manos con las palmas extendidas al cuerpo de los pacientes y realizan una "oración de intercesión". Ésta suele contener una invocación divina, la propiciación y apelación al poder del "Espíritu Santo", la exhortación de demonios y una serie de enunciados realizativos que marcan el sentido de la acción que "purga", "sana", "libera", "unge con el poder de Dios". Los registros audiovisuales que hemos producido de este momento muestran la correlación entre el discurso ritual y la emergencia de señales corporales en los fieles, entre las que se encuentran llantos, gemidos, modificaciones en la expresión facial, temblor de las extremidades superiores, sudoración. Este correlato somático se corrobora en el testimonio de los fieles que informan de sensaciones de escalofríos, carne de gallina, irradiación de calor o electricidad, experiencias de gozo y remisión de síntomas asociados a la imposición de manos, los que consideran "señales de la acción divina", cuyo poder "se derrama sobre los cuerpos".

La congregación proclama la "victoria del Espíritu sobre la carne" y celebra cantando los triunfos de la fe. Las manifestaciones somáticas, como las descritas, proveen una experiencia sensible del "Espíritu". La transformación de los estados 
afectivos en el culto, el acceso a estados alternativos de conciencia y el desplazamiento de síntomas, se valoran como obras de su magisterio sobrenatural. Estas vivencias se tienen como medio de legitimación de la doctrina, pruebas irrefutables de la verdad de la palabra, que conmina a los conversos a llevar una "vida en santidad". Quienes las han experimentado se consideran "sellados por el Espíritu".

Como consecuencia de este "magisterio de poder", se impone a los fieles el deber de seguir la ética pentecostal que, en el campo social, promueve valores como la austeridad, la fidelidad e indisolubilidad matrimonial y el respeto a las autoridades temporales en los planos político, laboral y familiar. Se trata de principios que resultan funcionales a la reproducción del orden establecido y que favorecen la integración de los fieles en una familia nuclear, su incorporación al mercado del trabajo y ciertos grados de acumulación económica (Moulian 2012). Estos cambios en la situación de los devotos son conocidos como "los frutos del Espíritu". No obstante, el análisis de las manifestaciones de la pneumatología en los cultos carismáticos nos muestra un segmento de participantes para los cuales las luchas no cejan, sino se reproducen de modo reiterativo. Los pentecostales califican estas situaciones que afectan a los creyentes como "pruebas" que Dios pone para evaluar su fidelidad. La enacción del Espíritu Santo se vuelve aquí un medio de compensación para sobrellevar la adversidad.

\section{Carisma, Compensación y Sobreadaptación}

La vida espiritual del ministerio evangelista que constituye nuestro principal caso de estudio ilustra, de modo ejemplar, esta circularidad en las manifestaciones del carisma. Aquí hemos conocido a un grupo de fieles asiduos al Espíritu, afectados por dramas que se muestran irresolubles, quienes buscan de manera periódica las manifestaciones de la presencia y las viven con particular intensidad. En medio de las cadenas terrenales "se gozan en la libertad del Espíritu". El pastor confirma esta relación entre aflicción y carisma. "Quien quiera trabajar en las cosas del Espíritu deberá soportar luchas y pruebas. Mientras más grandes sean las dificultades, mayores serán las bendiciones espirituales que recibirá", predica.

La variable de género resulta significativa en este contexto. Entre los agentes carismáticos destacan mujeres víctimas de violencia doméstica.
Encontramos un solo caso en el que el empoderamiento espiritual ha sido el medio para romper la relación tormentosa. Para la mayoría, el culto es el espacio donde encuentran fuerzas para sobrellevar el dolor y adaptarse a las condiciones de su vida cotidiana. El conservadurismo pentecostal en materia de relaciones parentales y de género, que defiende el matrimonio y la supeditación de la mujer al varón como principios de la moral cristiana, favorece la perpetuación de sistemas interaccionales familiares patológicos. Las mujeres entienden que ellos son las "pruebas" que deben enfrentar en sus vidas como signo de fidelidad a Dios.

En este contexto, aflicción y carisma muestran una estructura de retroalimentación doblevincular, es decir, de efectos contraproducentes, puesto que, a la vez que terapéuticos, resultan patogénicos. Así, la aflicción lleva al carisma y este a la aflicción, en tanto favorece la mantención de las condiciones que la generan. La "liberación espiritual" que ofrece el culto es el fundamento de un disciplinamiento social donde se reproducen las "cadenas" que conducen a los fieles a continuar refugiándose en el "Espíritu". Las funciones de la enacción del "Espíritu Santo" en el culto pentecostal se muestran, en este caso, contradictorias, pero integradas al servicio de este sistema religioso. Por una parte, provee una experiencia sensible de la presencia divina de cualidades transformadoras; por otra, es fuente de legitimación de una ética social quietista.

Quienes han experimentado en carne propia “el poder del Espíritu Santo" se ven conminados por esta evidencia a guardar una vida de fidelidad a Dios. En términos sociales, ello supone un alejamiento del mundo temporal para consagrarse a la vida de la Iglesia, la familia y el trabajo. Para muchos fieles ésta es una vía para salir de la marginalidad e integrarse en la sociedad. No obstante, la concepción providencionalista del orden establecido los hace conservadores incluso ante los componentes patológicos del mismo. Como hemos dicho, el culto se vuelve entonces doblevincular y sus funciones paradójicas, pues a la vez que ofrece a sus participantes una "liberación espiritual", ayuda a reproducir las condiciones que producen su malestar.

Las nociones de "luchas" y "pruebas" como designios en la vida del cristiano expresan esta actitud fatalista frente al mundo. El culto pentecostal provee los medios para administrar las situaciones de aflicción, pero no para combatir sus causas estructurales. Su ética social, en ocasiones, impone a 
los fieles la sobreadaptación a las mismas, es decir, una adaptación forzada a condiciones generadoras de frustración o dolor como, por ejemplo, sistemas de relaciones familiares patológicos. La enacción del "Espíritu Santo" sirve entonces como un espacio compensatorio y una vía de restauración para seguir "caminando entre luchas y pruebas".
Agradecimientos: Artículo escrito en el marco del proyecto Fondecyt 1090443, "Éxtasis del don y estigmas de posesión en el culto pentecostal", que ha hecho posible nuestro trabajo de investigación. A los evaluadores anónimos cuyas observaciones y sugerencias han contribuido a dar forma a la versión final de este texto.

\section{Referencias Citadas}

Alexander, B. 1989. Pentecostal ritual reconsidered: antiestructural dimension of possession. Journal of Ritual Studies $3: 109-128$

Bastian, J.P. 1997. La Mutación Religiosa de América Latina. Para una Sociología del Cambio en una Sociedad Periférica. Fondo de Cultura Económica, México.

Becker, J. 2005. Anthropological perspectives on music and emotion. En Music and Emotion: Theory and Research, editado por P. Juslin y J. Sloboda, pp. 135-160. Oxford University Press, Oxford.

Biblia 2003. Santa Biblia. Antiguo y Nuevo Testamento. Versión Reina Valera, revisada en 1960. Sociedades Bíblicas Unidas, Corea.

Bothner, M. 1994. El soplo del Espíritu: perspectivas sobre el movimiento pentecostal en Chile. Estudios Públicos 55:261-296.

Bourguignon, E. 1973. Religion, Altered States of Consciousness, and Social Change. Ohio University Press, Columbus.

Bruner, J. 1968. Processes of Cognitive Growth: Infancy. Clark University Press, Worcester.

Canales, M., P. Samuel y H. Villela 1991. En Tierra Extraña II. Para una Sociología de la Religiosidad Popular Protestante. Amerindia, Santiago.

Corvalán, O. 2009. Distribución, crecimiento y discriminación de los evangélicos pentecostales. Revista Cultura y Religión 3:76-98.

Chesnut, A. 1997. Born Again in Brazil: The Pentecostal Boom and the Pathogens of Poverty. Rutgers University Press, New Brunswick.

Foerster, R. 1993. Introducción a la Religiosidad Mapuche. Editorial Universitaria, Santiago.

Guerrero, B. 1994. A Dios Rogando. Pentecostales en la Sociedad Aymara del Norte Grande de Chile. Vrije Universiteit Press, Amsterdam.

Hollenweger, W. 1969. Pentecostalism and the Third World. Ecumenical Press Service 36:13-14.

Lalive D’Epinay, Ch. 1968. El Refugio de las Masas. Estudio Sociológico del Protestantismo Chileno. Editorial Pacífico, Santiago.

Lewis, I. 1989 [1971]. Ecstatic Religion. A Study of Shamanism and Spirit Possession. Second Edition, Routledge, London.
Martín Serrano, M. 1977. La Mediación Social .Akal, Madrid. Maturana, H. y F. Varela 1994 [1984]. El Árbol del Conocimiento. Undécima Edición, Editorial Universitaria, Santiago.

Merker, B., G. Madison y P. Ekckerdal 2009. On the role and origin of isochrony in human rhythmic entrainment. Cortex 45:4-17.

Mosher, R. 1995. Pentecostalism and Inculturation in Chile. Tesis doctoral. Pontificia Università Gregoriana, Vaticano.

Moulian, R. 2010. Dones espirituales y violencia de género en el culto pentecostal: emocionalidad, disociación y disciplinamiento. Ponencia presentada en el VII Congreso Chileno de Antropología, San Pedro de Atacama.

_ - _ 2012. Metamorfosis Ritual: desde el Ngillatun al Culto Pentecostal. Universidad Austral de Chile \& Kultrún, Valdivia.

Newberg, A., E. D"Aquili y V. Rause 2001. Why God Won"t Go Away: Brain Science and the Biology of Belief. Ballantine Books, New York.

Orellana, L. 2011. Hitos en la historia del movimiento pentecostal chileno. Ponencia presentada en el Congreso La Identidad del Pentecostalismo Latinoamericano. Red Latinoamericana de Estudios Pentecostales, Quito.

Ossa, M. 1991. Lo Ajeno y lo Propio. Identidad Pentecostal y Trabajo. Rehue, Santiago.

Parker, C. 1993. Otra Lógica en América Latina. Religión Popular y Modernización Capitalista. Fondo de Cultura Económica, Santiago.

Pike, K. 1967. Language in Relation to a Unified Theory of Structure of Human Behavior. The Hague, Mouton.

Poblete, R. y C. Galilea 1984. Movimiento Pentecostal e Iglesia Católica en Medios Populares. Bellarmino, Santiago.

Poewe, K. 1989. On the metonymic structure of religious experience: the example of charismatic Christianity. Cultural Dynamics 2:361-380.

Seligman, R. y L. Kirmayer 2008. Dissociative experience and cultural neuroscience: narrative, metaphor and mechanism. Culture, Medicine and Psychiatry 32:31-64.

Sjørup, L. 2002. Pentecostals: the power of the powerless. A Journal of Theology 41:16-25.

Spoerer, S. 1984. Las transformaciones del campo religioso en América Latina. Actas del Primer Congreso de Sociología Chilena, pp. 5-19. Academia de Humanismo Cristiano, Santiago. 
Tennekes, H. 1985. El Movimiento Pentecostal en la Sociedad Chilena. Sub-facultad de Antropología Cultural de la Universidad Libre de Amsterdam y Cuadernos de Investigación de la Universidad del Norte, Iquique.

Toro, S. 2011. La acción visible como texto relacional aprendido. Ponencia presentada en el VII Congreso Internacional Chileno de Semiótica, Valdivia.

Varela, F., E. Thompson y E. Rosch 1992. De Cuerpo Presente. Las Ciencias Cognitivas y la Experiencia Humana. Traducido por C. Gardini. Gedisa, Barcelona.
Varela, F. 2000. El Fenómeno de la Vida. Dolmen Ediciones, Santiago.

- _ _ 2002. Conocer. Las Ciencias Cognitivas: Tendencias y Perspectivas. Cartografía de las Ideas Actuales. Gedisa Editorial, Barcelona.

Willems, E. 1967. Followers of the New Faith: Culture and Rise of Pentecostalism in Brazil and Chile. Vanderbilt University Press, Nashville.

Zatorre, R., J. Chen y V. Penhune 2007. When the brain plays music: auditory-motor interactions in music perception and production. Nature Reviews, Neuroscience 8:547-558. 
